\title{
UNA DEFENSA DE LA PERSPECTIVA FORMATIVA DEL DESARROLLO PROFESIONAL DOCENTE EN LA PRÁCTICA EDUCATIVA DE LOS PROFESORES DE QUÍMICA
}

\author{
Ricardo Aponte-Buitrago* \\ Departamento de Química, Facultad de Ciencias, Universidad Antonio Nariño. Cra 3Este \# 47A-15, Bogotá, Colombia
}

Recebido em 29/05/2017; aceito em 05/09/2017; publicado na web em 27/10/2017

\begin{abstract}
A DEFENCE OF EDUCATIONAL PERSPECTIVE OF TEACHERS' PROFESSIONAL DEVELOPMENT IN EDUCATIONAL PRACTICE OF CHEMISTRY TEACHERS. Teachers' Professional Development (TPD) has been defined as learning opportunities, insights and discussions that improves the personal, social and pedagogical growing of the teachers, whose main goal is the enhancement of their knowledge about teaching strategies and research skills around educational practice. The above topics are needed to change the learning and teaching forms in chemical education; however, the tie between TPD and the classroom practice of the chemistry teachers still has not been greatly discussed in the literature. Thus, this paper seeks to generate a perspective upon theoretical issues related with TPD and their relevance in the chemistry teaching, where the teacher as researcher is put forward as a promoter of classroom research projects, which he joins chemical, pedagogical and practical knowledge for the improvement of learning process of his students. Moreover, the fruitful relationship between TPD and teaching of chemistry educators with the aim to make teachers' communities around their professional development is analyzed.
\end{abstract}

Keywords: Teachers' professional development; education in chemistry; teaching professional practice; teacher as researcher.

\section{INTRODUCCIÓN}

Una detallada revisión de la literatura sobre las construcciones teóricas y propuestas didácticas formuladas desde la modelización de conceptos químicos, que conllevan al mejoramiento en las formas de enseñanza y aprendizaje de la química ${ }^{1,2}$ o sobre las tensiones pedagógicas de los profesores en esta área del conocimiento en su práctica educativa, ${ }^{3}$ muestran que las reflexiones en torno a su Desarrollo Profesional Docente (DPD) han sido escasamente discutidas; ${ }^{4-7}$ no obstante, las relaciones entre la formación inicial y continuada de profesores de química en el proceso de autorregulación de su DPD, en la búsqueda de su identidad como educador y en la consolidación de su práctica profesional, así como el vínculo que tiene en la estructura y finalidades de dichos programas de formación, son aspectos que se han mencionado aisladamente en algunos textos..$^{8-11}$

La corta discusión alrededor del DPD en los profesores de química ha generado como consecuencia educadores ajenos a las necesidades educativas, individuales, sociales y culturales de los estudiantes y las instituciones donde ejercen su acción pedagógica y didáctica, especialmente en la educación universitaria, puesto que la estrúctura rígida y lineal de los currículos en los cursos y programas de formación basados en métodos aprobados por políticas educativas gubernamentales, buscan en el profesor universitario de química (y en todos los profesores), un modelo de profesionalismo docente basado en la realización de su "mejor práctica" donde, coaccionados por la normatividad educativa, no dispongan de habilidades para elaborar otros modelos profesionales por sí mismos. ${ }^{12}$

En esta medida, el profesor de química no será quien resuelva problemas pedagógicos en su práctica profesional, sino un técnico que con su discurso y habilidades específicas pretenda la producción de algún aprendizaje en sus estudiantes; así, el DPD en el profesor como técnico, es un entrenamiento que busca la consolidación de algunas habilidades para la adquisición de ciertos niveles de experticia. Para ello, los cursos cortos de formación docente o la asistencia a eventos programados por agentes educativos expertos que

*e-mail: ricardoaponte@uan.edu.co guíen a los educadores al cumplimiento de las políticas educativas estatales, son suficientes para el enriquecimiento de su desarrollo profesional, aunque nada de lo mencionado represente correctamente el significado de la profesión docente. ${ }^{13,14}$

Por otra parte, los insuficientes fundamentos teóricos que sustentan las ideas epistemológicas de los profesores formadores y en formación, las orientaciones problemáticas en su formación centrada solo en los contenidos o solo en aspectos psico-pedagógicos, y la realidad actual del contexto profesional, donde los profesores se interesan más por generar acciones en y para los estudiantes de forma pasiva, más no con ellos, contribuyen de manera acelerada al desinterés en una formación universitaria que responda integralmente a las necesidades educativas contemporáneas. ${ }^{12,15}$

En efecto, estas problemáticas han forjado actualmente tres barreras difíciles de franquear, particularmente en los profesores de química a nivel universitario: 1. La química debe ser enseñada por profesionales que tengan experiencia y dominio del conocimiento científico químico sin preponderancia de su formación didáctica y pedagógica, 2. La enseñanza de los conceptos químicos se basa en la transmisión inequívoca de los modelos científicos eruditos, haciendo énfasis en su complejidad matemática y subestimando cualquier aproximación didáctica analógica que conduzca a errores conceptuales en los estudiantes y 3. Una formación a través de cursos, talleres y diplomados es suficiente para fortalecer los aspectos epistemológicos, didácticos y curriculares propios de la enseñanza de la química y favorecer sus modelos de profesionalización y DPD.

Aunque hay investigaciones extensas y bien fundamentadas que analizan y establecen la ineficacia, la ambigüedad y las dificultades que conduce la enseñanza de los modelos científicos en el aula sin una adecuación hacia los modelos didácticos analógicos o modelos científicos escolares, ${ }^{16-18}$ dichas discusiones aún siguen siendo extrañas y difusas en la practica docente de los profesores en química en todos los niveles de formación.

Así, la presente discusión en torno al DPD como eje coyuntural en el cuerpo conceptual y profesional de los profesores de química, tiene como objetivo responder tres preguntas que surgen a partir de las dificultades anteriormente expuestas: 1. ¿Qué tipo de formación 
pedagógica y didáctica es necesaria y suficiente en los profesores para enseñar química?, 2. ¿Qué modelo de DPD es adecuado para generar un modelo fuerte de profesionalización docente? y 3. ¿Dónde está y hacia dónde va el DPD en su formación y ejercicio profesional?

\section{¿Qué se conoce acerca del DPD?}

Resolver cada una de las preguntas anteriormente expuestas exige un conocimiento profundo tanto de los fundamentos teóricos y metodológicos del desarrollo profesional docente como de los modelos que lo conforman, ${ }^{19}$ los cuales incluyen diversas relaciones entre las distintas dimensiones que forman integralmente a un profesor: personal, pedagógica y epistemológica, social y cultural, como se presenta en la Figura 1.



Figura 1. Relaciones entre el DPD y las dimensiones del profesor
Actualmente, las publicaciones en torno al mejoramiento de las formas de enseñanza y aprendizaje de conceptos químicos a través de estrategias didácticas basadas en la modelización, las actitudes de los maestros, las políticas educativas, el análisis del discurso docente, entre otros, han tratado aspectos relacionados con su desarrollo profesional. Pero, ¿qué es el DPD? Algunos autores lo han definido como una oportunidad de aprendizaje, como un proceso que transforma el conocimiento docente o aquel que favorece la autorregulación metacognitiva del profesor, pero esta diversidad depende de la perspectiva que se tenga frente a su formación y práctica (Tabla 1).

Estas concepciones acerca del DPD en distintos ámbitos de la práctica educativa, permite referir el desarrollo profesional para los profesores de química como el conjunto de reflexiones, discusiones y aprendizajes que favorecen continuamente su crecimiento personal, social, cultural y profesional desde aspectos pedagógicos, didácticos y epistemológicos, los cuales generan actitudes y aptitudes de indagación crítica y transformación efectiva en sus formas de enseñanza y aprendizaje, tanto de los modelos científicos químicos como de los modelos escolares construídos a partir de la investigación en el aula.

Todo esto conlleva a que el profesor de química promueva en su quehacer el análisis permanente de su práctica educativa, reconozca los contextos espaciales y temporales dónde lleva a cabo su ejercicio profesional y renueve su compromiso como agente de cambio en el mejoramiento de la calidad educativa, teniendo en cuenta la revisión constante, planificada, autorregulada y consciente (individual y cooperativamente con otros profesores), del desarrollo emocional y cognitivo de su estudiantes y de sí mismo.

\section{EI DPD y la práctica docente del profesorado}

Así, dos ideas bien arraigadas en el pensamiento del profesor pueden discutirse con base en las formulaciones anteriores: en primer

Tabla 1. Algunas definiciones del Desarrollo Profesional Docente

Año publicación y página $\quad$ Definición de DPD

2002:663

2003:12

2004:220

2007:78

2009:55

2011:116

2013:165

2014:108

2015:214

2017:219
Oportunidades de aprendizaje en las que participan docentes creativos y reflexivos en vía de fortalecer sus prácticas que independientemente de su actividad presenta tres criterios: lectura, investigación-acción y escritura de reflexiones. ${ }^{20}$

Proceso a largo plazo que incluye oportunidades y experiencias regulares planeadas sistemáticamente para promover crecimiento y desarrollo en la profesión..$^{21}$

Proceso de aprendizaje resultante de la interacción significativa con el contexto (espacial y temporal), que eventualmente conduce a cambios en la práctica profesional de los profesores (acción) y en el pensamiento acerca de su práctica. ${ }^{22}$

Proceso presente a lo largo de toda la vida de un profesor, que incluyen tensiones personales relacionadas con su voluntad de aprender y tensiones externas proporcionadas por las actividades de formación organizadas y su relación con las necesidades de los sistemas educativos a la que pertenecen los profesores. ${ }^{23}$

Serie de procesos de autorregulación metacognitiva que llevan a un crecimiento en los ámbitos que orientan la profesión docente como consecuencia de la comprensión, la práctica y la relación entre pensar, sentir y hacer en el aula y en la institución. Todos estos ámbitos hacen parte de la dimensión individual del profesor que se ve influenciado por el ámbito externo. ${ }^{24}$

Uso de aprendizajes formales e informarles que profundizan y entienden las competencias profesionales de los profesores, incluyendo su conocimiento, creencias, motivaciones y habilidades de autorregulación. ${ }^{25}$

Proceso de apoyo, seguimiento y acompañamiento que incide en el desarrollo profesional y personal del docente, reconociéndolo no como un funcionario de la enseñanza o experto disciplinar, sino como un verdadero profesional de la enseñanza universitária. ${ }^{26}$

Aprendizaje que busca generar en el docente una postura de reflexión y transformación de su práctica, adquiriendo así una actitud indagatoria frente a la enseñanza. De esta manera, el profesor es un aprendiz que recoge y analiza evidencias acerca de sus prácticas y el efecto que éstas tienen sobre sus alumnos. ${ }^{5}$

Proceso de aprendizaje basado en la reflexión, que posibilita la revisión y exploración de las experiencias y el conocimiento, dando lugar a nuevas comprensiones y por ende, a nuevas acciones en el ejercicio docente. ${ }^{6}$

Proceso evolutivo que se construye a medida que los profesores adquieren experiencia, sabiduría y conciencia profesional. También es continuo por el desarrollo de concepciones epistemológicas y prácticas profesionales provenientes de experiencias formales e informales, adquiridas a lo largo de la vida escolar y social que pueden ser perfeccionadas a lo largo de su carrera. ${ }^{7}$ 
lugar, el docente no puede prescindir de los contextos educativos sobre los cuales ejerce su acción pedagógica, ${ }^{27}$ dado que su desarrollo profesional no debe sujetarse exclusivamente a la formación en un determinado campo disciplinar, subestimando la formación en modelos pedagógicos y didácticos.

Si bien el conocimiento base (disciplinar) y práctico (acciones que realiza en el contexto educativo) son fundamentales para la profesionalización docente, la construcción de subsídios teóricos derivados de la pedagógica y la didáctica evitan el ejercicio basado en el modelo de transmisión - repetición, así como la replicación de recetas, aunando todos los esfuerzos en formas de enseñanza y aprendizaje significativas de los conceptos químicos.

Sin embargo, una formación enfocada únicamente en los aspectos pedagógicos y didácticos propios de la química sin reconocimiento e indagación sobre su conocimiento base también es perjudicial, puesto que la débil formación en su campo disciplinar hará que el profesor presente problemas en la selección de ideas centrales en torno a los conceptos químicos, en la construcción de modelos teóricos frente a los mismos y además desconozca las dificultades asociadas a su enseñanza, así como las consecuencias erróneas que generan en su aprendizaje. $^{28}$

Sumado a ello, algunas investigaciones han concluido que el conocimiento pedagógico y didáctico aprendido en la formación inicial presenta una escasa o nula relación con el ejercicio docente efectuado en el aula; ${ }^{15}$ esto conduce a una realidad en la cual las formas de enseñanza utilizadas por los docentes se basen en la imitación de aquellos profesores que generaron un impacto positivo en su formación personal o en aquellos que determinaron su orientación profesional como educador.

Desde luego, la conclusión pareciera dirigirse hacia el establecimiento de una relación entre los dos tipos de conocimiento sin preponderancia alguna; no obstante, la reflexión debe ir más allá y buscar que el docente tenga un papel más activo en el diseño, desarrollo y evaluación de estrategias de intervención didáctica, así como en la formulación y ejecución de proyectos que favorezcan los procesos de enseñanza y aprendizaje en el aula. En este sentido, el Conocimiento Pedagógico del Contenido (CPC) ha realizado valiosos aportes dentro de campos del conocimiento científico químico altamente especializados. ${ }^{26-28}$

De esta forma, el profesor toma distancia frente a la adquisición acelerada de conocimientos en su disciplina, así como de rutinas didácticas aprendidas a lo largo de cursos cortos de formación y se transforma consecuentemente en un gestor, ejecutor y evaluador de proyectos de innovación e investigación didáctica. En efecto, la formación y el desarrollo profesional del profesorado migra del mundo de los comportamientos y los acontecimientos escolares al mundo de los significados y sentidos en torno a su profesión. ${ }^{27}$

Estos cambios en las formas de pensamiento del profesor permiten el paso del modelo débil al modelo fuerte de profesionalización docente, donde éste no es un simple técnico que se somete a las directrices de los lineamientos pedagógicos, metodológicos y curriculares propuestos por las políticas educativas, sin espacio para la discusión y el desarrollo de nuevas iniciativas en el aula, sino un solucionador de problemas pedagógicos cuyo desarrollo profesional se cimenta en la investigación educativa. En este sentido, el profesor puede tomar decisiones entre la teoría y la práctica cuando relaciona el conocimiento investigativo con las evidencias obtenidas en las actividades realizadas en el aula (Figura 2).

En un segundo lugar y no menos importante, la intención del docente no es suficiente para efectuar cambios significativos en su práctica pedagógica ni promover nuevos desafíos en su desarrollo profesional; ${ }^{29}$ frente a ello es necesario recalcar que los cambios en las prácticas docentes no derivan de modificaciones efectuadas individualmente en torno a sus prácticas, en la ampliación de recursos didácticos o en la adquisición y profundización en nuevos conceptos propios de la disciplina.

Realizar cambios en el ejercicio profesional docente requiere una revisión de las prácticas desde el análisis del discurso, la conversación con otros profesores, la observación de clases, la participación en talleres o cursos y la formación de redes de trabajo que deriven en comunidades de profesores en pro del mejoramiento de su aprendizaje y desarrollo profesional. ${ }^{7,25,30}$

De esta manera, encontramos dos polos bien fundamentados en torno al DPD, a saber: el factor personal, referido a la voluntad que tiene el profesor como profesional capaz de aprender, basado en el compromiso moral con sus estudiantes y con la institución sobre la que ejerce su práctica y el factor contextual, relacionado con las necesidades de los sistemas y las políticas educativas, las actividades de formación y las oportunidades de aprendizaje. La tensión entre estos dos aspectos sigue definiendo los programas de formación inicial, continuada y los modelos de desarrollo y aprendizaje profesional docente. ${ }^{7,23}$



Figura 2. Relaciones entre modelos débil y fuerte para la profesionalización docente 
Este último factor puede analizarse en detalle con base en la clasificacion de las oportunidades de aprendizaje que sustentan al DPD en oportunidades formales (o tradicionales), basadas en la formación pedagógica y didáctica a partir de cursos de actualización docente y talleres que deben desarrollar los educadores a lo largo de un determinado tiempo para el mejoramiento de sus habilidades profesionales, o en oportunidades informales que se nutren de las observaciones de las clases, la socialización de experiencias exitosas en el aula, la elaboración de comunidades de aprendizaje entre docentes o las asesorías con especialistas que orienten el mejoramiento de su actividad. ${ }^{21,25,30}$ Pero, ¿Qué factores, modelos y oportunidades de aprendizaje son importantes para mejorar el DPD en los profesores de química?

\section{Una propuesta formativa del DPD para el profesorado de química}

Antes de defender una propuesta para el DPD en el profesorado de química, es necesario resaltar cuatro aspectos que generan problemas en los docentes durante su práctica educativa:

1. Los profesores dan mayor importancia al conocimiento científico químico que al conocimiento pedagógico y desconocen los avances relacionados con el CPC.

2. Los docentes cuya formación inicial se ha llevado a cabo estrictamente en el campo de la química (Químicos o Licenciados en Química) subestiman a aquellos cuya formación se ha enfocado en aspectos pedagógicos y disciplinares (Licenciados en Educación en Química), pormenorizando su saber pedagógico.

3. El pensamiento del profesor de química se orienta hacia una versión a-histórica y epistemológicamente positivista del conocimiento científico químico, donde se busca que el estudiante conozca los modelos propios de los científicos y reproduzca experimentos de manera repetitiva con el objetivo de comprender su complejidad.

4. La formación por medio de cursos cortos, seminarios o talleres realizados de modo aislado, es suficiente para cubrir la demanda pedagógica requerida en su ejercicio docente; por tanto, el profesor ideal de química será aquel que teniendo un conocimiento base profundo en su disciplina, apenas lo complemente con un vago y desarticulado saber pedagógico y didáctico.

Así mismo, otros argumentos tales como el desinterés del profesor por el contexto educativo que lo rodea y el recelo por su práctica, evidenciado por la incomodidad que genera la crítica de algún otro docente a sus metodologías, demandan la búsqueda de soluciones para estas dificultades en su ejercicio docente; en efecto, el primer aspecto a reflexionar en torno a estas problemáticas debe relacionarse con los objetivos que debe trazarse el DPD para los profesores de química, sin que lo posteriormente presentado se vea como una "receta" a cumplir, debido a que el DPD en los profesores de química depende de su desarrollo personal, profesional y social, completamente único y diferente en cada educador.

En esta medida, la Figura 3 presenta unos posibles objetivos y sus respectivas estrategias de desarrollo para el DPD en los docentes de química, que si bien se han discutido para los programas de formación docente, son útiles para plantear las finalidades en su práctica educativa.

Basado en este direccionamiento, la percepción de la práctica docente cambia en el profesor, quien deja de ser un instructor y se transforma en un investigador de su práctica (PI), cuyo cambio está sujeto no sólo a variaciones en su modelo de profesionalización, donde el PI es un solucionador de problemas pedagógicos en el aula, sino también al paso de un modelo de Desarrollo a uno de Aprendizaje profesional para el mejoramiento de su labor educativa. ${ }^{12,13,31} \mathrm{La}$ Tabla 2 expone las características que presentaría este cambio de modelo específicamente para la práctica pedagógica de los docentes de química.

La tabla anterior no indica, sin embargo, que referirse al desarrollo profesional docente sea por lo tanto errado tanto en su denotación como en su connotación dentro de la práctica educativa de un profesor de química; lo que esto conlleva es a pensar el DPD como un modelo de aprendizaje profesional a través del cual construye su propio discurso y sus presupuestos teóricos en torno a la práctica educativa, de manera autónoma frente a planes de estudio rígidos y descontextualizados de la dinámica escolar.

Este modelo de aprendizaje está mucho más relacionado con la naturaleza de la ciencia, en tanto que es tentativa y dependiente de la observación, la reflexión teórica y el análisis; además, promueve de manera eficaz la apertura a nuevas formas de pensamento. ${ }^{12}$

Sin embargo, el aprendizaje profesional debe ser congruente con los presupuestos que consolidan el estatuto conceptual del desarrollo profesional para la formación docente, ${ }^{30}$ aplicable también para su ejercicio, el cual consta de cinco etapas: el enfoque en contenidos, el aprendizaje activo, la coherencia, la duración y la participación colectiva (Figura 4).

De esta forma, la figura anterior ofrece al profesor investigador un marco de referencia para indagarse en cada uno de los aspectos que conforman el núcleo conceptual del DPD y definir si su quehacer

\section{Objetivos del DPD}

Establecer relaciones entre el conocimiento base y pedagógico en los profesores de química a través de la formación docente, la generación de redes académicas y la creación y ejecución de proyectos de innovación e investigación en el aula.

Objetivos especificos

1. Comprender el impacto de los contextos escolares en la formación y ejercicio de los docentes.

2. Construir relaciones profesionales a partir de la discusión de sus prácticas con pares académicos.

3. Formular y ejecutar proyectos curriculares y de investigación escolar que cimenten significados y sentidos y frente a su ejercicio profesional.



y la reflexión sobre sus prácticas. 
Tabla 2. Características de los modelos de DPD en los profesores de química

\begin{tabular}{|c|c|c|}
\hline \multirow{2}{*}{ Característica } & \multicolumn{2}{|c|}{ Modelo } \\
\hline & Desarrollo Profesional & Aprendizaje Profesional \\
\hline Profesionalización docente & Basado en el modelo débil de profesor visto como instructor. & $\begin{array}{l}\text { Basado en el modelo fuerte de profesor visto como } \\
\text { investigador. }\end{array}$ \\
\hline Práctica educativa & $\begin{array}{l}\text { Búsqueda permanente de la mejor práctica que cumpla con } \\
\text { los lineamientos educativos propuestos por las políticas } \\
\text { educativas. }\end{array}$ & $\begin{array}{l}\text { Construcción colectiva y reflexiva de la práctica apropiada a } \\
\text { través de la investigación en el aula. }\end{array}$ \\
\hline Desarrollo personal & $\begin{array}{l}\text { Se basa en las necesidades particulares de los profesores y en } \\
\text { sus requerimientos individuales donde se consideran únicos } \\
\text { agentes de transformación en el aula. }\end{array}$ & $\begin{array}{l}\text { Busca el compartimiento de prácticas como soluciones a sus } \\
\text { problemas pedagógicos. }\end{array}$ \\
\hline Desarrollo social & $\begin{array}{l}\text { Promueve el desarrollo de su práctica desde el aislamiento y } \\
\text { el recelo, asumiendo una posición defensiva ante la crítica de } \\
\text { sus pares. Introspección y búsqueda de una zona de confort } \\
\text { construida a partir de un pequeño conjunto de acciones } \\
\text { efectivas en el aula. }\end{array}$ & $\begin{array}{l}\text { Construye y favorece comunidades de aprendizaje } \\
\text { que permitan la investigación, la reflexión, crítica y } \\
\text { retroalimentación de su ejercicio profesional basado en el } \\
\text { trabajo colaborativo. }\end{array}$ \\
\hline $\begin{array}{l}\text { Desarrollo profesional: } \\
\text { formación }\end{array}$ & $\begin{array}{l}\text { Basada en contenidos propios de la química sin articulación } \\
\text { con el conocimiento pedagógico. El DPD se resume en la } \\
\text { adquisición de habilidades y herramientas para efectuar las } \\
\text { mejores prácticas a través de cursos de actualización docente. }\end{array}$ & $\begin{array}{l}\text { Prevalece la articulación entre el conocimiento químico, } \\
\text { didáctico y pedagógico para la reformulación del conocimiento } \\
\text { profesional y práctico que mejore las prácticas educativas. La } \\
\text { química vista como ciencia escolar. }\end{array}$ \\
\hline $\begin{array}{l}\text { Desarrollo profesional: práctica } \\
\text { educativa }\end{array}$ & $\begin{array}{l}\text { Se basa en lineamientos impuestos por las políticas educativas } \\
\text { y libros de texto que no son coherentes con la realidad de los } \\
\text { estudiantes y las instituciones educativas. Se siguen al pie de } \\
\text { la letra planes y programas exitosos en otros países, sin su } \\
\text { respectiva adecuación y análisis de fortalezas y dificultades. }\end{array}$ & $\begin{array}{l}\text { Se desarrolla con base en el cambio y la innovación en las } \\
\text { prácticas a través del trabajo con pares para desarrollar } \\
\text { actitudes, habilidades y conocimiento coherente con objetivos } \\
\text { comunes. Los profesores efectúan una teoría de su propia } \\
\text { práctica que no se enriquece con el entrenamiento sino con } \\
\text { la labor docente en sí misma. }\end{array}$ \\
\hline Aspectos curriculares & $\begin{array}{l}\text { Currículos rígidos, basados en la acumulación de contenidos } \\
\text { y en el cumplimiento de estándares de calidad impuestos por } \\
\text { las políticas educativas, sin el análisis del contexto educativo } \\
\text { y del desarrollo actitudinal, aptitudinal y cognitivo de sus } \\
\text { estudiantes. }\end{array}$ & $\begin{array}{l}\text { Currículos flexibles basados en el análisis epistemológico } \\
\text { e histórico de los conceptos químicos y en la modelización } \\
\text { como eje transversal para la representación de estos. El } \\
\text { diseño curricular no se ajusta a contenidos sino a objetivos } \\
\text { de enseñanza y aprendizaje basados en la química aplicada y } \\
\text { las relaciones ciencia, tecnología y sociedad. }{ }^{32}\end{array}$ \\
\hline Evaluación de su práctica & $\begin{array}{l}\text { Se evalúa según el número de cosas que se hagan para, sobre } \\
\text { o en los estudiantes desde una perspectiva pasiva que limite } \\
\text { las oportunidades conjuntas de aprendizaje. }\end{array}$ & $\begin{array}{l}\text { Se fundamenta en el conjunto de acciones que se efectúan en el } \\
\text { aula junto con estudiantes y profesores, con el fin de alcanzar } \\
\text { objetivos compartidos en torno a la solución de problemas } \\
\text { pedagógicos propios. }\end{array}$ \\
\hline
\end{tabular}

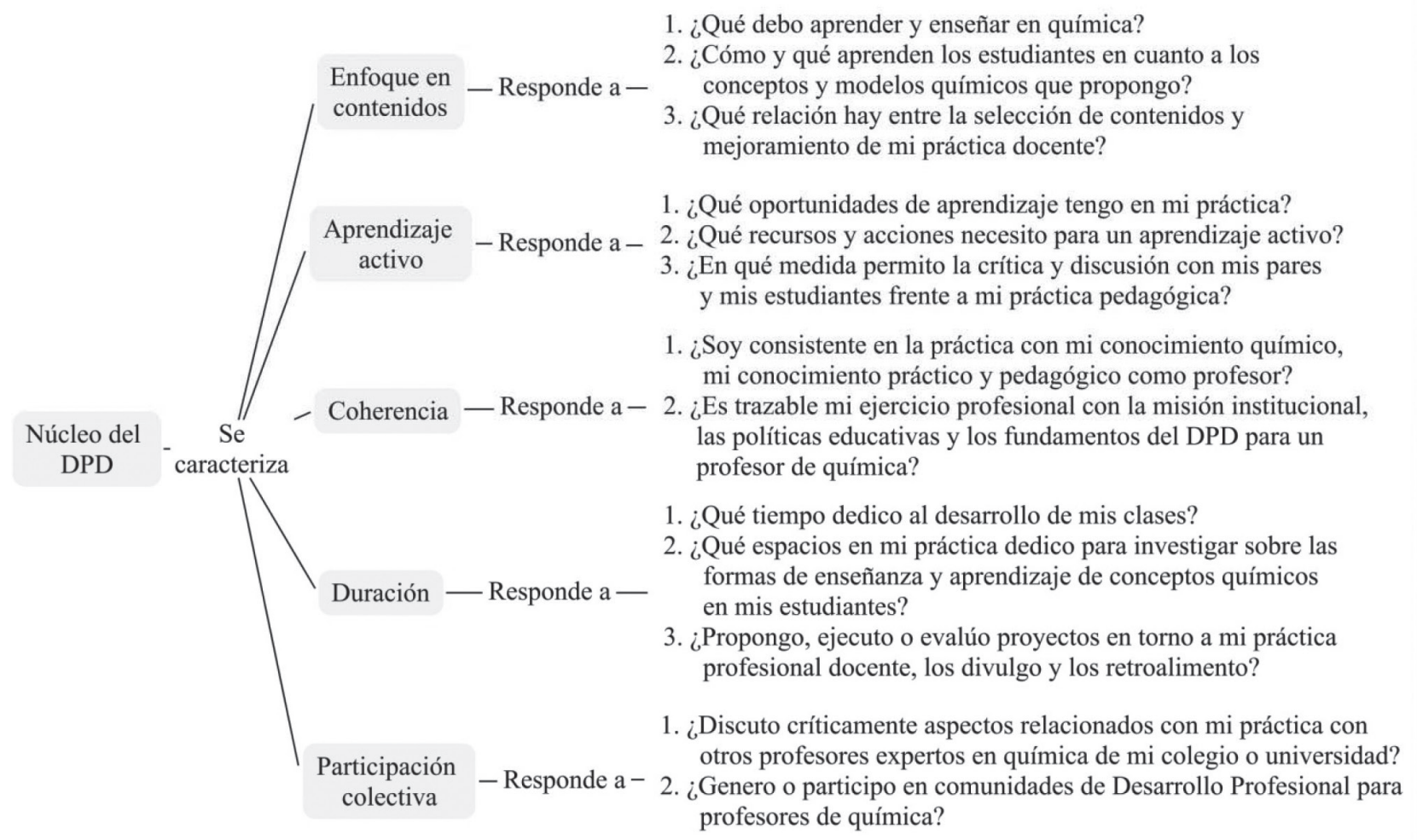

Figura 4. Una propuesta sobre el estatuto del DPD para la práctica de los profesores de química 
está enmarcado en alguna de estas etapas. ${ }^{25,29}$ Con esta propuesta, se pretende que el profesor de química no sea un reproductor de modelos científicos, sino un planeador de proyectos de innovación en torno a la construcción y consolidación de modelos científicos escolares, favoreciendo oportunidades informales de aprendizaje en el aula.

Este cambio de perspectiva debe permear en su discurso profesional, generar alternativas en sus formas de enseñanza desde presupuestos constructivistas y convertir al docente en un investigador en el aula que diseña proyectos basados en la modelización de conceptos químicos, contextualizados con su realidad educativa. ${ }^{21,24,33}$

El hecho de que el enfoque en contenidos incluya la formación inicial y continuada de profesores a través de su participación en especializaciones, maestrías, doctorados, congresos y cursos cortos en pedagogía y didáctica, transversales en la estructuración de su conocimiento profesional, no conlleva a que dichos espacios se asuman como añadiduras usadas para transmitir su conocimiento científico químico de un modo repetitivo y para justificar sus errores en el conocimiento práctico, definido éste como las acciones que el profesor lleva a cabo en su práctica pedagógica guiadas por sus actitudes, sus habilidades y su relación con el contexto escolar. ${ }^{34}$

En este punto, los PI son profesores expertos, en tanto que tienen un compromiso con su formación en áreas específicas del conocimiento químico y didáctico de la química, y establecen relaciones que favorezcan los procesos de enseñanza y aprendizaje de una forma responsable.

En consecuencia, el PI construye un solo engranaje entre los conocimientos disciplinar, profesional y práctico en el aula por medio de investigaciones y reflexiones críticas sobre su quehacer, analizando con sus pares experiencias exitosas que enriquezcan su desarrollo profesional y vinculando estos elementos con transformaciones significativas en su discurso, con el mejoramiento de su práctica educativa, con la autoconfianza en sus habilidades reflexivas y oportunidades informales de aprendizaje que, de forma contextualizada con el entorno escolar y congruente con las políticas educativas, le brinden autonomía para el desarrollo de actividades innovadoras en el aula (Figura 5).

Así, estas interrelaciones buscan evitar lo que es un hecho dentro del ejercicio profesional docente en química, evidente aún más en la educación universitaria: el profesor más ocupado de los contenidos que de las formas de enseñanza y aprendizaje, la supeditación del conocimiento práctico al conocimiento disciplinar y la consideración del conocimiento pedagógico como un conjunto de metodologías y estrategias aisladas que facilitan la enseñanza de un concepto químico.

Este último se basa en la replicación de prácticas exitosas realizadas por sus maestros, la imitación de las metodologías y actitudes de otros "profesores modelo", la aplicación de discusiones alrededor de aspectos pedagógicos, didácticos y filosóficos propios de la química sin ningún tipo de lectura inferencial y posterior adaptación, quizás aprendidos en algún curso o seminario y la promoción del aprendizaje individual y repetitivo de términos, que predomina sobre otras formas de carácter constructivista. ${ }^{21,34}$

El PI en química, por tanto, debe encontrar en todas las etapas del $\mathrm{DPD}^{29}$ una coyuntura para reflexionar sobre su práctica bajo distintas estrategias como la modelización de conceptos químicos, necesario para modificar sus formas de enseñanza, la deriva de oportunidades informales de aprendizaje sobre estos y el análisis de su discurso basado en ideas centrales propias de la química y no en conceptos carentes de un núcleo epistemológico que soporte la justificación de sus enunciados. ${ }^{35}$

Así mismo, el profesor de química debe repensar su quehacer en el aula, las representaciones y estructuras semánticas (mapas conceptuales, mentefactos, esquemas, etc.) que desarrolla para el aprendizaje y la enseñanza de una determinada idea relevante en la estructura conceptual de la química, y además retroalimentar su conocimiento base, profesional y práctico con sus estudiantes, pares o con PI expertos que apoyen estos procesos ${ }^{23}$ (Figura 6).

Aunque el establecimiento de un marco de referencia para el DPD en cada etapa es dispendioso pues depende del desarrollo personal, cultural, social y profesional de cada profesor, es necesario



Figura 5. Una propuesta de modelo relacional entre la formación del PI en química y su DPD 


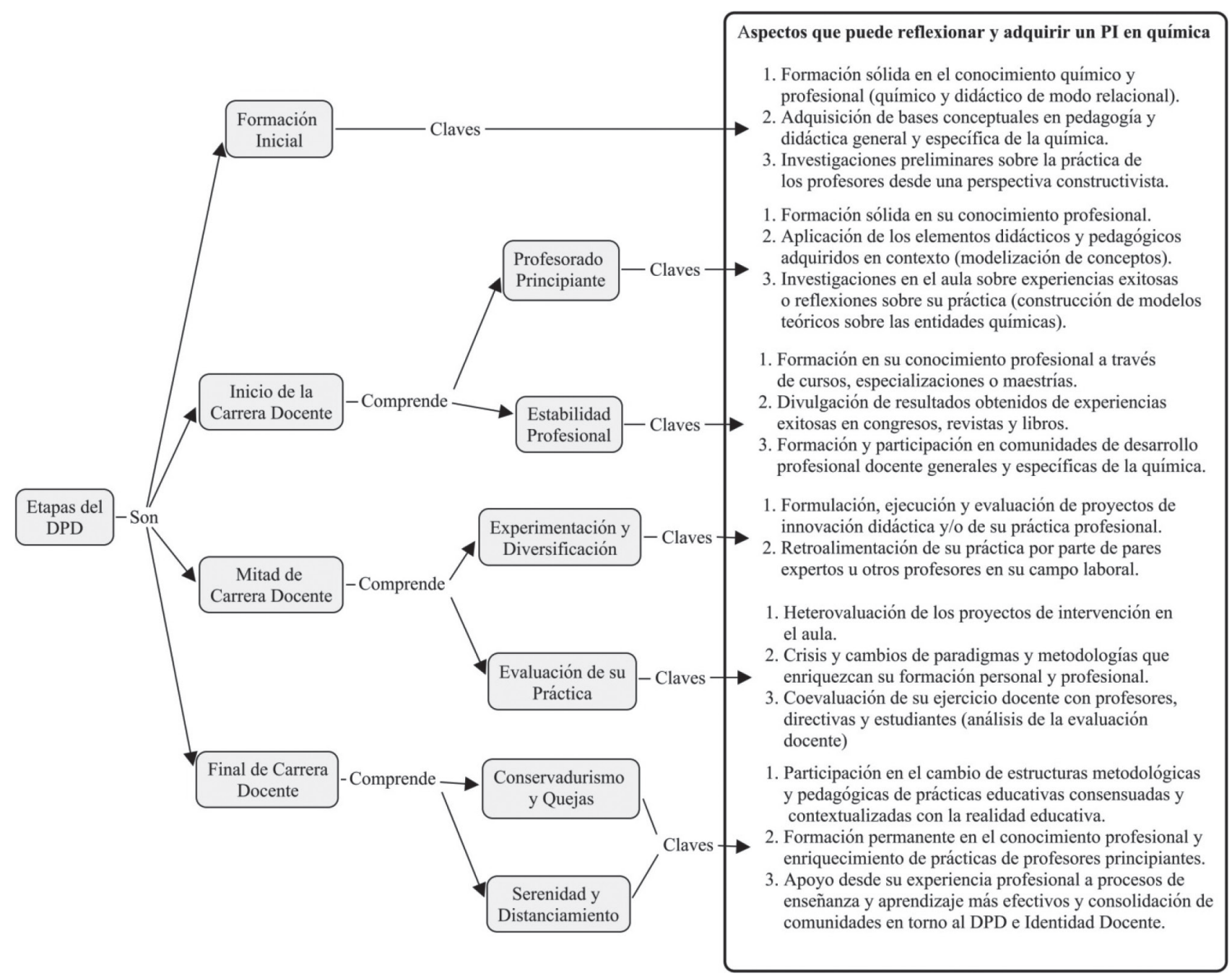

Figura 6. Modelo de reflexión del PI en las distintas etapas del DPD $D^{25,29}$

remarcar que las claves presentadas sirven como puntos de partida para la reflexión en torno a la formación y la práctica educativa del profesorado en química y permite reformular nuevas perspectivas frente a un aprendizaje profesional significativo.

Los nuevos desafíos en torno al DPD van más allá de conseguir una receta o fórmula definitiva para el desarrollo profesional en los profesores de química, lo cual es imposible de cumplir. De tal modo, estos subsidios teóricos están direccionados hacia la búsqueda de nuevas perspectivas en torno al rol del profesor investigador en el aula, a la reflexión alrededor de modelos de desarrollo profesional y profesionalización docente y a repensar en la necesidad de promover en los profesores de química investigadores educativos, con base en el trabajo colaborativo, la innovación en su práctica y en la pesquisa de un modelo fuerte que consolide su profesión docente.

Solo en esta medida, el profesor será visto como un sujeto que aprende y genera sus propios modelos escolares, como un profesional íntegro en el conocimiento químico, práctico y profesional, que relaciona su formación disciplinar con la didáctica y pedagógica aprendida no sólo en cursos, maestrías o doctorados, sino aquella que adquiere en su quehacer permeado por la innovación, la autonomía y el trabajo colaborativo con otros profesores y, ante todo, como un PI en el aula que genera nuevos proyectos curriculares, habilidades y conocimientos.

Así, el profesor de química será reconocido como una persona a la que debe evaluarse solamente por su labor educativa, como un sujeto con capacidad crítica y reflexiva frente a su quehacer, que forma y se forma junto con profesores, directivos y estudiantes a través de la innovación, la autonomía y la investigación y, en definitiva, como un profesional en el aula. ${ }^{24,34}$

\section{CONSIDERACIONES FINALES}

Frente a las preguntas que orientaron esta discusión, es posible presentar algunas consideraciones a saber: No hay una formación necesaria y tampoco suficiente que supla definitivamente las formas de enseñanza y aprendizaje de la química. El PI debe estar siempre a la vanguardia y analizar críticamente los avances en cuanto a su campo disciplinar y didáctico, que consolidan la estructura conceptual de la química, divulgados por medio de reflexiones y experiencias exitosas. 2. No debe existir una preponderancia del saber disciplinar sobre el pedagógico y viceversa, pues el DPD en el PI en química se constituye como un vínculo entre su formación disciplinar, pedagógica y sus habilidades investigativas.

Así, el Desarrollo Profesional de un profesor de química no debe depender únicamente de un tipo de conocimiento, sino de las relaciones que pueda generar con otros saberes, a fin de mejorar sus prácticas en el aula, los procesos de aprendizaje en sus estudiantes, sus actitudes, sus habilidades indagatorias y críticas frente a su quehacer a través de proyectos de intervención en la enseñanza, los currículos y, finalmente, frente a los significados y sentidos en torno a su profesión docente.

Por último, bastante se ha discutido sobre el DPD de manera 
general para todos los campos disciplinares y por ello se ha generado una gran número de reflexiones e investigaciones sobre el tema; sin embargo, en química se ha escrito poco al respecto, tal vez por la importancia o la urgencia de abordar otros elementos que directamente impactan en el desarrollo profesional de un profesor de química como la modelización, las estrategias didácticas para la enseñanza de un concepto o la revisión de ideas previas y errores conceptuales frente a un tema.

Sin embargo, no podemos olvidar que el conocimiento científico químico es un pretexto en la relación pedagógica presente en el aula, pues esta última no es más que una interacción entre personas y la profesión docente, humanizada y humanizante, debe tener en cuenta que el profesor es un poderoso agente que, se pretenda o no, actúa sobre las actitudes de sus estudiantes determinando la naturaleza de su estructura académica, contribuyendo en la construcción de su sistema social y estableciendo los ritmos y los cambios en los diferentes procesos de enseñanza y aprendizaje. ${ }^{27}$

En definitiva, aunque el DPD en los docentes de química aún está madurando y crece velozmente con la implementación de nuevas estrategias y reflexiones pedagógicas, didácticas, filosóficas y epistemológicas propias de la estructura conceptual de la química, se necesitan articular todos estos elementos con el fin de hacer del DPD un modelo de aprendizaje profesional que impacte en el desarrollo social, personal y profesional del profesor y favorezca además, un modelo fuerte de profesionalización docente que se fundamente en la investigación educativa y el discurso sobre la práctica reflexiva en el aula. ${ }^{13,14}$

De este modo, el DPD hará parte del estilo de vida del profesor en su formación y ejercicio, cuya base se cimentará en las transformaciones permanentes de perspectivas y construcciones teóricas con base en nuevas oportunidades informales de aprendizaje adquiridas en su estudio o en su práctica educativa. Pero, más allá de indagar ¿Hacia dónde va el DPD?, la verdadera pregunta es: ¿En qué modelo de desarrollo profesional estamos?

\section{AGRADECIMIENTOS}

A los pares revisores de este artículo por sus valiosos aportes en la consolidación de la discusión presentada.

\section{REFERENCIAS}

1. Giudice, J.; Galagovsky, L. En Didáctica de las Ciencias Naturales. El caso de los modelos científicos; Galagovsky, L., ed.; Lugar Editorial: Buenos Aires, 2011, cap. 8.

2. Marchán-Carvajal, I.; Sanmartí, N.; Educ. Quim. 2015, 26, 267.

3. Rius, P.; Educ. Quim. 2011, 22, 123.

4. Peres, F.; Marques, C.; Delizoicov, D.; Revista Brasileira de Pesquisa em Educação em Ciências 2007, 7, 1.

5. González-Weil, C.; Gómez, M.; Ahumada, G.; Bravo, P.; Salinas, E.; Avilés, D.; Pérez, J.; Santana, J.; Estudios Pedagógicos 2014, 40, 105.

6. Marzábal, A.; Rocha, A.; Toledo, B.; Educ. Quim. 2015, 26, 212.
7. Passos, C.; Del-Pino, J.; Quim. Nova 2017, 40, 219.

8. Gallego-Badillo, R.; Pérez-Miranda, R.; Enseñanza de las Ciencias 2002, 20, 401.

9. Gallego-Badillo, R.; Pérez-Miranda, R.; Torres-de-Gallego, L.; GallegoTorres, A.; Revista Electrónica de Enseñanza de las Ciencias 2006, 5, 481.

10. Gallego-Badillo, R.; Pérez-Miranda, R.; Torres-de-Gallego, L.; Ciência \& Educação 2004, 10, 219.

11. Rocha, A.; Bertelle, A.; Iturralde, C.; García de Cajén, S.; Roa, M.; Fuhr, A.; Boucíguez, M.; Revista Eureka sobre Enseñanza y Divulgación de las Ciencias 2013, 10, 836.

12. Smith, C.; Svendsen, B.; Gray, P. En Science Education. An International Course Companion; Taber, K., Akpan, B., eds.; Sense Publishers: Rotterdam, 2017, cap. 40.

13. Taber, K.; Classroom-based Research and Evidence-based Practice. A student's guide, SAGE Publications: Trowbridge, 2007.

14. Gilbert, J.; Int. J. Sci. Educ. 1994, 16, 511.

15. Furió-Más, C.; Gil-Pérez, D.; Enseñanza de las Ciencias 1989, 7, 257.

16. Galagosky, L.; Aduriz-Bravo, A.; Enseñanza de las Ciencias 2001, 19, 231.

17. Aduriz-Bravo, A.; Izquierdo, M.; Revista Electrónica de Investigación en Educación en Ciencias 2009, 4, 40.

18. Chamizo, J.; Science \& Education 2013, 22, 1613.

19. Bell, B.; Gilbert, J.; Teaching and Teacher Education 1994, 10, 483

20. Bredeson, P.; International Journal of Educational Research 2002, 37, 661.

21. Villegas-Reimers, E.; Teacher professional development: an international review of the literature. IIEP: Paris, 2003.

22. Kelchtermans, G. En International Handbook on the Continuing Professional Development of Teachers; Sachs, J.; Day, C., eds.; McGraw-Hill Education: Glasgow, 2004, cap. 9.

23. Avalos, B.; Pensamiento Educativo 2007, 41, 77.

24. García-Martínez, A.; Tecné, Episteme y Didaxis 2009, 26, 53.

25. Richter, D.; Kunter, M.; Klusmann, U.; Lüdtke, O.; Baumert, J.; Teaching and Teacher Education 2011, 27, 116.

26. Londoño-Orozco, G.; Revista de la Universidad de la Salle 2013, 57, 161.

27. Lorenzo-Vicente, J.; Revista Complutense de Educación 1998, 9, 141.

28. Talanquer, V.; Educ. Quim. 2004, 15, 60.

29. Álvarez-Álvarez, C.; Enseñanza y Desarrollo Profesional Docente. La Muralla: Madrid, 2013.

30. Desimone, L.; Educational Researcher 2009, 38, 181.

31. Borko, H.; Educational Researcher 2004, 33, 3.

32. Caamaño, A. En Investigar en la enseñanza de la química. Nuevos horizontes: contextualizar y modelizar; Izquierdo M.; Caamaño, A.; Quintanilla, M., eds.; Universitat Autònoma de Barcelona: Bellatera, 2007, cap. 1.

33. Stolk, M.; Bulte, A.; de Jong, O.; Pilot, A.; Chem. Educ. Res. Pract. 2009, 10, 164.

34. Muñoz-Martínez, M.; Garay-Garay, F.; Estudios Pedagógicos 2015, 41, 389.

35. Talanquer, V.; J. Chem. Educ. 2016, 93, 3. 